\title{
Advanced Breast Carcinoma
}

National Cancer Institute

\section{Source}

National Cancer Institute. Advanced Breast Carcinoma. NCI Thesaurus. Code C162648.

Breast carcinoma that has spread extensively to other anatomic sites or is no longer responding to treatment. 